\title{
CPTT: A High-throughput Coding-aware Routing Metric for Multi-hop Wireless Networks
}

\author{
Hao Yue ${ }^{\dagger}$, Xiaoyan Zhu*, Chi Zhang ${ }^{\S}$ and Yuguang Fang ${ }^{\dagger}$ \\ ${ }^{\dagger}$ Department of Electrical and Computer Engineering, University of Florida, Gainesville, FL 32611 \\ *National Key Laboratory of Integrated Services Networks, Xidian University, Xi'an, China \\ ${ }^{\S}$ School of Information Science and Technology, University of Science and Technology of China, Hefei, China \\ Email: hyue@ufl.edu,xyzhu@mail.xidian.edu.cn, chizhang@ustc.edu.cn, fang@ece.ufl.edu
}

\begin{abstract}
Network coding is widely recognized as a promising approach to increase the throughput of wireless networks. In order to maximize the benefit of network coding, the consideration of potential coding opportunities is incorporated into the route selection, which is referred to as coding-aware routing. Most of existing coding-aware routing metrics are designed based on traditional routing metrics like expected transmission count (ETX) and fail to take many critical factors into account, such as traffic load, link transmission rate and interference. Therefore, the routes discovered with them are always sub-optimal. In this paper, we present a novel routing metric called Coding-aware Path Transmission Time (CPTT). CPTT considers the effect of traffic load, multirate, intra-flow and inter-flow interference as well as network coding and quantifies them in a unified manner, which can be used to accurately evaluate path performance and discover the path with high throughput. Through extensive simulations, we compare CPTT with different coding-aware routing metrics proposed in the literature and show that the paths selected with CPTT have maximum end-to-end throughput under network coding.
\end{abstract}

\section{INTRODUCTION}

First proposed by Ahlswede et al. [1], network coding is becoming an emerging communication paradigm to improve the performance of wireless networks. Exploiting the inherent broadcast nature of wireless medium, network coding can deliver multiple packets in a single transmission, and thus yields much higher throughput. This has been proved from theoretical study to practical implementations. Liu et al. in [2] and Le et al. in [3] provide the upper bounds on the throughput gain achieved by network coding. COPE [4], the first applicable network coding system for multi-hop wireless networks, is shown to be capable of achieving variable coding gain up to $400 \%$ in different topologies and traffic loads.

Since network coding could significantly increase the network throughput, routing with awareness of potential coding opportunities has been investigated by many researchers, which is referred to as coding-aware routing [5]. Based on the expected transmission count (ETX) routing metric, two coding-aware routing metrics, expected transmission count with coding awareness (ETX-CA) [9] and expected resource consumption (ERC) [7], are proposed, in which the benefit

This work was supported in part by the U.S. National Science Foundation under grant CNS-1147813. The work of C. Zhang was partially supported by the National Natural Science Foundation of China under grant 61202140. of network coding is counted as the decrease in the number of transmissions to deliver a packet along a path. But due to the simpleness of ETX, neither of them take traffic load and link transmission rate into account, which are two important factors that affect the path throughput. In [6], Le et al. introduce a new coding-aware routing metric called CRM. CRM calculates the total number of transmissions to deliver the packets in the buffers of all relay nodes on a route with network coding, which jointly considers traffic load, interference and coding opportunities and facilitates the performance comparison between coding-possible and codingimpossible paths. Under CRM as well as ETX-AC and ERC, the transmission cost of a path is simply modeled as the sum of all the link cost. However, this additivity actually does not hold in wireless networks, where the links can transmit simultaneously when they are sufficiently far apart from each other. Thus, these existing routing metrics cannot accurately reflect the performance of a path and the paths selected based on them are always sub-optimal in terms of end-to-end throughput.

We have already designed a routing metric in [10] to effectively evaluate the performance of a path when there is only one flow in a network. In this paper, we extend it into multi-flow scenario and incorporate the effect of network coding into it. The main contributions of this paper are listed below:

- We design a novel routing metric for path selection with network coding, which is called Coding-aware Path Transmission Time (CPTT). CPTT captures the effect of traffic load, multirate, transmission interference as well as network coding on pact capacity and accurately reflects the path performance.

- Extensive simulations show that CPTT outperforms various existing coding-aware routing metrics and always discoveries paths with the highest throughput under network coding.

The rest of this paper is organized as follows. Section II introduces the network model. In Section III and IV, we propose the coding-aware routing metric CPTT. We conduct numerical simulations and compare the performance of different coding-aware routing metrics in Section V. Finally, we 
draw the concluding remarks in Section VI.

\section{PRELIMINARIES}

\section{A. Network Model}

Consider a multi-hop wireless network with a collection of static nodes. Each node is equipped with an omni-directional antenna for communications, and can only transmit or receive at one time to prevent self-interference at the physical layer. There are a set of unicast flows in the network. Each flow follows a single path from the source to the destination. We assume a scheduling-based MAC layer in which the time domain is divided into timeslots with the equal constant duration.

\section{B. Network Coding}

Network coding can be classified into two categories: interflow network coding and intra-flow network coding. In this paper, we concentrate on inter-flow network coding. Assume $n$ flows $f_{1}, \ldots, f_{n}$ cross at one node and the intersection node has $n$ packets, $p_{1}, \ldots, p_{n}$, from the $n$ flows to forward to different nexthops, $r_{1}, \ldots, r_{n}$. With inter-flow network coding, instead of transmitting the $n$ packets $p_{1}, \ldots, p_{n}$ individually, the intersection node can XOR them together and broadcast only the encoded packet to all the nexthops if and only if each next-hop $r_{i}$ already has all $n-1$ packets $p_{j}$ for $j \neq i$, which could happen when either of the conditions below is satisfied for each next-hop $r_{i}$ :

- $r_{i}$ is the one-hop predecessors of the intersection node on the path of flow $f_{j}$;

- $r_{i}$ is in the neighborhood of the one-hop predecessors of the intersection node along the path of flow $f_{j}$ and has overheard packet $p_{j}$ from its transmission.

The above conditions can be used to identify the potential coding opportunities in a network. Fig. 1 shows two typical examples of such coding opportunities.

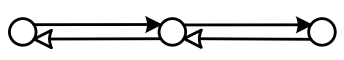

(a) Alice-and-Bob topology

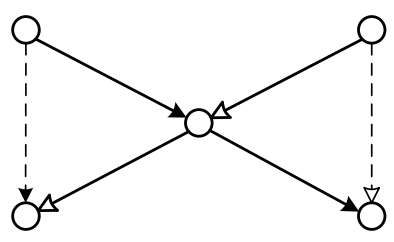

(b) "X" topololy
Fig. 1. Two examples of coding opportunities.

\section{iII. Path Transmission Time}

In this section, we present a new coding-blind routing metric with consideration of traffic load, multirate and transmission interference, which serves as the basis for the design of our coding-aware routing metric.

\section{A. Link Conflict Graph}

We use a communication graph $\mathcal{G}=(\mathcal{V}, \mathcal{E})$ to represent the wireless network. Here, each vertex $v \in \mathcal{V}$ corresponds to a node in the network, and there is a directed wireless link $l=(i, j) \in \mathcal{E}$ if node $j$ is within the communication range of node $i$. Let $s_{l}$ and $d_{l}$ stand for the sender and the receiver of link $l=(i, j)$, respectively, i.e., $s_{l}=i$ and $d_{l}=j$.

Transmission interference can be described by different models. In this paper, we consider the widely used protocol model [11]. With protocol model, any other transmitter has to be at least a certain distance away from an ongoing receiver to avoid interference. Let $I_{i}$ be the interference range of node $i$. Let $D_{i j}$ denote the distance between nodes $i$ and $j$. Then, two links $l_{1}=(i, j)$ and $l_{2}=(u, v)$ interfere with each other if either $D_{i v} \leq I_{i}$ or $D_{u j} \leq I_{u}$. In order to describe the contention relationship of all the links in the network, a link conflict graph $\mathcal{U}=(\mathcal{E}, \mathcal{I})$ can be constructed, whose vertices denote the wireless links and each edge represents the conflict relationship between two different links. A clique $\mathcal{C} \subseteq \mathcal{E}$ is a set of links, any two of which cannot transmit simultaneously without interference. If $\mathcal{C}$ is no longer a clique after adding any one more link, $\mathcal{C}$ is a maximal clique.

\section{B. Path Transmission Time}

We first define a new quantity called the clique transmission time $t_{\mathcal{C}}$ of a clique $\mathcal{C}$ as follows:

$$
t_{\mathcal{C}}=\sum_{l: l \in \mathcal{C}} \frac{f_{l}}{r_{l}}
$$

where $r_{l}$ denotes the transmission rate of link $l$ and $f_{l}$ represents the total traffic rate of all the flows in the network that travel through link $l$. Since at any time only one link in a clique can be scheduled, the clique transmission time $t_{\mathcal{C}}$ indicates the accumulated fraction of time to finish the traffic delivery of every link in the clique.

Assume there already exist several flows in the network. Now a new flow $\pi$ is injected into the network with traffic rate $x$ and a candidate path $\mathcal{P}$. For each link $l$ along path $\mathcal{P}$, let $\mathscr{C}_{l}$ represent the set of all the cliques that contain the link $l$, i.e., $\mathscr{C}_{l}=\{\mathcal{C} \mid l \in \mathcal{C}\}$. Then, the transmission time $t_{l}$ of all the traffic over link $l$ under interference from other links satisfies:

$$
t_{l} \geq \max _{\mathcal{C}: \mathcal{C} \in \mathscr{C}_{l}} t_{\mathcal{C}}
$$

Thus, the upper bound of the capacity of link $l$ for flow $\pi$ can be calculated as

$$
c_{l} \leq \frac{x}{t_{l}}=\frac{x}{\max _{\mathcal{C}: \mathcal{C} \in \mathscr{C}_{l}} t_{\mathcal{C}}} .
$$

Since the performance of a path is primarily determined by the bottleneck link, the throughput of path $\mathcal{P}$ for flow $\pi$ is upper bounded by

$$
c_{\mathcal{P}} \leq \min _{l: l \in \mathcal{P}} c_{l} \leq \min _{l: l \in \mathcal{P}}\left(\frac{x}{\max _{\mathcal{C}: \mathcal{C} \in \mathscr{C}_{l}} t_{\mathcal{C}}}\right)=\frac{x}{\min _{l: l \in \mathcal{P}}\left(\max _{\mathcal{C}: \mathcal{C} \in \mathscr{C}_{l}} t_{\mathcal{C}}\right)}
$$


Eqn. (4) shows that $\min _{l: l \in \mathcal{P}}\left(\max _{\mathcal{C}: \mathcal{C} \in \mathscr{C}_{l}} t_{\mathcal{C}}\right)$ is inversely proportional to the upper bound of path capacity. Therefore, it has the capability to measure the throughput of different paths. We adopt it as our new routing metric, which is called Path Transmission Time (PTT), i.e.,

$$
\operatorname{PTT}(\mathcal{P})=\min _{l: l \in \mathcal{P}}\left(\max _{\mathcal{C}: \mathcal{C} \in \mathscr{C}_{l}} t_{\mathcal{C}}\right) .
$$

Given a set of candidate paths for a certain flow, the one with the minimum value of PTT potentially has the optimal throughput.

\section{Coding-aware Path Transmission Time}

Network coding exploits the broadcast nature of wireless medium to transmit multiple packets simultaneously over a number of links and thus increases the network throughput. In this section, we incorporate the effect of network coding into the metric PTT and design a new coding-aware routing metric.

\section{A. Hyperlink Conflict Graph}

To model both the multicast transmissions of the encoded packets from one node to several nexthops and the general unicast packet delivery in a unified manner, we define a hyperlink as a collection of links from the same sender to a non-empty set of receivers. For a hyperlink $\mathcal{L}$, if $|\mathcal{L}|>1$, it indicates the multicast transmission over the links in $\mathcal{L}$ with network coding. Otherwise, it represents unicast transmissions. Similarly, the sender and receivers of hyperlink $\mathcal{L}$ are denoted by $s_{\mathcal{L}}$ and $d_{\mathcal{L}}$, respectively. Then, the wireless network can also be represented as a hyperlink communication graph $\mathcal{G}^{*}=$ $\left(\mathcal{V}, \mathcal{E}^{*}\right)$, where $\mathcal{V}$ denotes the set of nodes and $\mathcal{E}^{*}$ is the set of hyperlinks.

Each hyperlink $\mathcal{L}$ is associated with a transmission rate $r_{\mathcal{L}}$, which equals to the minimum transmission rate of the links in the hyperlink to guarantee packet reception at all receivers, i.e.,

$$
r_{\mathcal{L}}=\min _{l: l \in \mathcal{L}} r_{l}
$$

Based on the protocol model, the hyperlink conflict graph $\mathcal{U}^{*}=\left(\mathcal{E}^{*}, \mathcal{I}^{*}\right)$ can be created as the extension of the link conflict graph $\mathcal{U}=(\mathcal{E}, \mathcal{I})$. Each vertex in $\mathcal{E}^{*}$ represents a hyperlink $\mathcal{L}$ in the hyperlink communication graph. Two hyperlinks $\mathcal{L}_{1}$ and $\mathcal{L}_{2}$ contend with each other and hence there is an edge between them in $\mathcal{U}^{*}$ if either

- There exists one node $j \in d_{\mathcal{L}_{1}}$, such that $D_{j s_{\mathcal{L}_{2}}} \leq I_{s_{\mathcal{L}_{2}}}$, or

- There exists one node $j \in d_{\mathcal{L}_{2}}$, such that $D_{j s_{\mathcal{L}_{1}}} \leq I_{s_{\mathcal{L}_{1}}}$.

A hyperlink clique $\mathcal{C}^{*} \subseteq \mathcal{E}^{*}$ consists a collection of hyperlinks, which mutually interfere with each other and at most one of them can transmit at any time. Fig. 2 illustrates the " $\mathrm{X}$ " topology and its hyperlink conflict graph, where $\mathcal{L}_{1}=\left\{l_{1}\right\}$, $\mathcal{L}_{2}=\left\{l_{2}\right\}, \mathcal{L}_{3}=\left\{l_{3}\right\}, \mathcal{L}_{4}=\left\{l_{4}\right\}$ and $\mathcal{L}_{5}=\left\{l_{1}, l_{2}\right\}$.

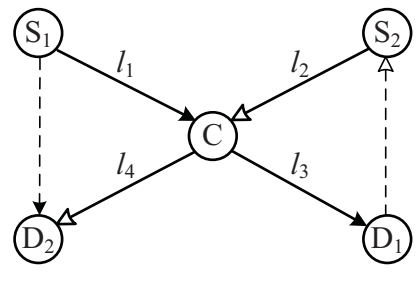

(a)

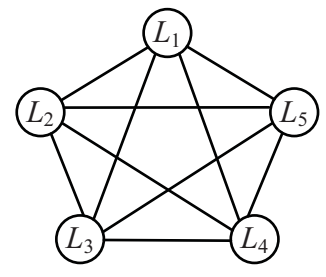

(b)
Fig. 2. The "X" topology and its hyperlink conflict graph.

\section{B. Coding-aware Path Transmission Time}

For ease of presentation, we abuse the notation a little bit without causing confusion. Given a link $l$ in the communication graph $\mathcal{G}=(\mathcal{V}, \mathcal{E})$ and a hyperlink clique $\mathcal{C}^{*}$, we say that $l \in \mathcal{C}^{*}$ if there exists a hyperlink $\mathcal{L} \in \mathcal{C}^{*}$ such that $l \in \mathcal{L}$. Let $\mathscr{L}_{\mathcal{C}^{*}}$ represent the set of all the links included in $\mathcal{C}^{*}$, i.e., $\mathscr{L}_{\mathcal{C}^{*}}=\left\{l \mid l \in \mathcal{C}^{*}\right\}$.

Considering network coding, the traffic over one link in the network might be transmitted by unicasting or by multicasting. Therefore, the computation of clique transmission time $t_{\mathcal{C}^{*}}$ is much more complicated than that without network coding, which could be calculated in the following way:

$$
\begin{aligned}
& \operatorname{Min} t_{\mathcal{C}^{*}} \\
\text { s.t. } \quad t_{\mathcal{C}^{*}} & =\sum_{\mathcal{L}: \mathcal{L} \in \mathcal{C}^{*}} w_{\mathcal{L}} \\
f_{l} & \leq \sum_{\mathcal{L}: \mathcal{L} \in \mathcal{C}^{*}} w_{\mathcal{L}} R_{l \mathcal{L}} \quad\left(\forall l \in \mathscr{L}_{\mathcal{C}^{*}}\right)
\end{aligned}
$$

where $w_{\mathcal{L}}$ denote the transmission time of hyperlink $\mathcal{L}$ and $R_{l \mathcal{L}}$ is the effective rate on link $l$ if hyperlink $\mathcal{L}$ is activated, i.e.,

$$
R_{l \mathcal{L}}= \begin{cases}r_{\mathcal{L}} & \text { If } l \in \mathcal{L} \\ 0 & \text { otherwise }\end{cases}
$$

Let $\mathscr{C}_{l}^{*}$ denote the set of all the hyperlink cliques that contain link $l$, i.e., $\mathscr{C}_{l}^{*}=\left\{\mathcal{C}^{*} \mid l \in \mathcal{C}^{*}\right\}$. Based on different transmission strategies, $\mathscr{C}_{l}^{*}$ can be further divided into two complementary subsets. Let $\mathscr{C}_{l}^{n c}$ denote the set of hyperlink cliques existing when the traffic over link $l$ is transmitted by multicasting with network coding, i.e., $\mathscr{C}_{l}^{n c}=\left\{\mathcal{C}^{*} \mid \mathcal{C}^{*} \in\right.$ $\mathscr{C}_{1}^{*}$ and there exists $\mathcal{L} \in \mathcal{C}^{*},|\mathcal{L}|>1$ such that $\left.l \in \mathcal{L}\right\}$. Let $\mathscr{C}_{l}^{u}=\mathscr{C}_{l}^{*} \backslash \mathscr{C}_{l}^{n c}$, which consists of all the hyperlink cliques discovered when the traffic on link $l$ is delivered only by unicasting without network coding. For example, the set $\mathscr{C}_{l_{3}}^{*}$ includes all the hyperlink cliques containing link $l_{3}$ in Fig. 2, such as $\left\{\mathcal{L}_{3}, \mathcal{L}_{4}, \mathcal{L}_{5}\right\}$ and $\left\{\mathcal{L}_{3}, \mathcal{L}_{4}\right\}$. We have $\left\{\mathcal{L}_{3}, \mathcal{L}_{4}, \mathcal{L}_{5}\right\} \in$ $\mathscr{C}_{l_{3}}^{n c}$ since $l_{3} \in \mathcal{L}_{5}$ and $\left|\mathcal{L}_{5}\right|=2$. For the hyperlink clique $\left\{\mathcal{L}_{3}, \mathcal{L}_{4}\right\}$, it is obvious that $\left\{\mathcal{L}_{3}, \mathcal{L}_{4}\right\} \in \mathscr{C}_{l}^{u}$.

We first consider the links with network coding opportunities. For such an link $l$ on path $\mathcal{P}$, if the coding opportunities are exploited, the traffic will be multicasted with network coding and the link transmission time $t_{l}^{n c}$ is lower bounded by 


$$
t_{l}^{n c} \geq \max _{\mathcal{C}^{*}: \mathcal{C}^{*} \in \mathscr{C}_{l}^{n c}} t_{\mathcal{C}^{*}}
$$

Otherwise, the traffic on link $l$ is transmitted only by unicasting and the link transmission time $t_{l}^{u}$ is lower bounded by

$$
t_{l}^{u} \geq \max _{\mathcal{C}^{*}: \mathcal{C}^{*} \in \mathscr{C}_{l}^{u}} t_{\mathcal{C}^{*}}
$$

The link transmission times $t_{l}^{n c}$ and $t_{l}^{u}$ indicate the capacity of link $l$ for flow $\pi$ under different transmission strategies, with or without network coding. Certainly, the one with minimum transmission time will be selected. Therefore, when network coding is available over link $l$, the time for the traffic over link $l$ to be transmitted satisfies

$$
t_{l}=\min \left\{t_{l}^{n c}, t_{l}^{u}\right\} \geq \min \left\{\max _{\mathcal{C}^{*}: \mathcal{C}^{*} \in \mathscr{C}_{l}^{n c}} t_{\mathcal{C}^{*},} \max _{\mathcal{C}^{*}: \mathcal{C}^{*} \in \mathscr{C}_{l}^{u}} t_{\mathcal{C}^{*}}\right\} .
$$

If there is no coding opportunity available for link $l$, it will only involve in unicast transmissions. We have $\mathscr{C}_{l}^{n c}=\emptyset$, $\mathscr{C}_{l}^{u}=\mathscr{C}_{l}^{*}$ and

$$
t_{l}=t_{l}^{u} \geq \min \left(\max _{\mathcal{C}^{*}: \mathcal{C}^{*} \in \mathscr{C}_{l}^{u}} t_{\mathcal{C}^{*}}\right)
$$

In this case, we set $\max _{\mathcal{C}^{*}: \mathcal{C}^{*} \in \mathscr{C}_{l}{ }^{n c}} t_{\mathcal{C}^{*}}=+\infty$ in Eqn. (11), which will make it equivalent to Eqn. (12). In this way, we can simply use Eqn. (11) to represent the transmission time of link $l$ under two scenarios in a unified manner.

Based on Eqn. (11), the throughput of the selected path $\mathcal{P}$ for flow $\pi$ with consideration of network coding is upper bounded by

$$
\begin{aligned}
& c_{\mathcal{P}} \leq \min _{l: l \in \mathcal{P}} c_{l} \\
& =\min _{l: l \in \mathcal{P}}\left(\frac{x}{\min \left\{t_{l}^{n c}, t_{l}^{u}\right\}}\right) \\
& \leq \frac{x}{\min _{l: l \in \mathcal{P}}\left(\min \left\{\max _{\mathcal{C}^{*}: \mathcal{C}^{*} \in \mathscr{C}_{l}{ }^{n c}} t_{\mathcal{C}^{*}}, \max _{\mathcal{C}^{*}: \mathcal{C}^{*} \in \mathscr{C}_{l}^{u}} t_{\mathcal{C}^{*}}\right\}\right)}
\end{aligned}
$$

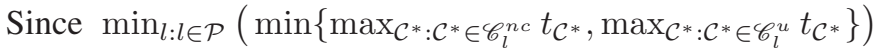
is able to reflect the path performance, we define it as the new routing metric, which is called coding-aware path transmission time (CPTT):

$$
C \operatorname{PTT}(\mathcal{P})=\min _{l: l \in \mathcal{P}}\left(\min \left\{\max _{\mathcal{C}^{*}: \mathcal{C}^{*} \in \mathscr{C}_{l}{ }^{n \mathcal{C}}} t_{\mathcal{C}^{*}}, \max _{\mathcal{C}^{*}: \mathcal{C}^{*} \in \mathscr{C}_{l}{ }^{u}} t_{\mathcal{C}^{*}}\right\}\right) .
$$

Note that if no network coding opportunity exists in the whole network, all the hyperlinks in the hyperlink communication graph $\mathcal{G}^{*}=\left(\mathcal{V}, \mathcal{E}^{*}\right)$ only contain one link in the communication graph $\mathcal{G}=(\mathcal{V}, \mathcal{E})$. In that case, Eqn (14) will reduce to

$$
\begin{aligned}
C P T T(\mathcal{P}) & =\min _{l: l \in \mathcal{P}}\left(\min \left\{\max _{\mathcal{C}^{*}: \mathcal{C}^{*} \in \mathscr{C}_{l}{ }^{n c}} t_{\mathcal{C}^{*},}, \max _{\mathcal{C}^{*}: \mathcal{C}^{*} \in \mathscr{C}_{l}{ }_{l}} t_{\mathcal{C}^{*}}\right\}\right) \\
& =\min _{l: l \in \mathcal{P}}\left(\max _{\mathcal{C}^{*}: \mathcal{C}^{*} \in \mathscr{C}_{l}{ }_{l}} t_{\mathcal{C}^{*}}\right) \\
& =\min _{l: l \in \mathcal{P}}\left(\max _{\mathcal{C}: \mathcal{C} \in \mathscr{C}_{l}} t_{\mathcal{C}}\right) \\
& =P T T(\mathcal{P})
\end{aligned}
$$

which indicates that the metric PTT defined in Section III-B is a special case of the CPTT without awareness of network coding.

\section{Performance Evaluation}

In this section, we evaluate the performance of the following coding-aware routing metrics on illustrative and random topologies: (1) ETX+CA; (2) CRM and (3) CPTT. In [13], Sengupta et al. have derived a mathematical formulation for computing path throughput with network coding under a scheduling-based MAC, which is used here to calculate the throughput of different paths discovered with those codingaware routing metrics.

\section{A. Evaluation on Illustrative Topology}

We start with an 13-node illustrative topology shown in Fig. 3. We assume all the wireless links in the network have the same transmission rate, which is equal to 1 unit per second. The interference range of each node is two-hop long. There already exists one flow from node 1 to node 2 with path $1 \rightarrow 5 \rightarrow 2$. The traffic rate is 5 units per second. Now multiple flows from node 3 to node 7 are continuously injected into the network, each of which has the traffic rate of 1 unit per second. There are two alternative paths to be chosen for these flows, which are illustrated in Fig. 3. Apparently, path 1 will create a coding opportunity at node 5 , which can be utilized to increase the throughput. The advantage of path 2 is less interference since it is far away from the other two routes. Next, we compare the throughput of the paths selected by the above coding-aware routing metrics for the first 12 flows from node 3 to node 7 .

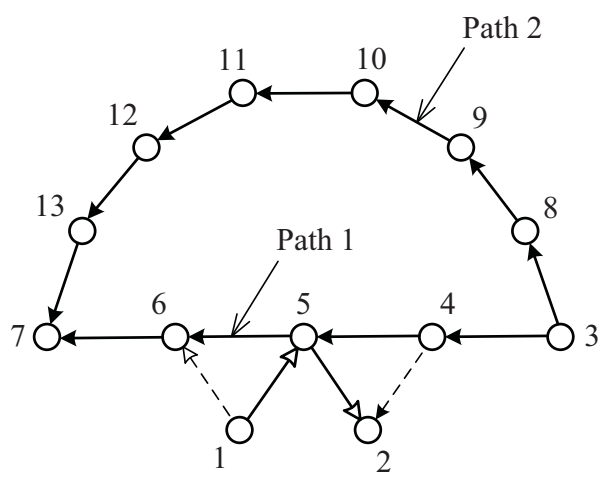

Fig. 3. The illustrative topology. 


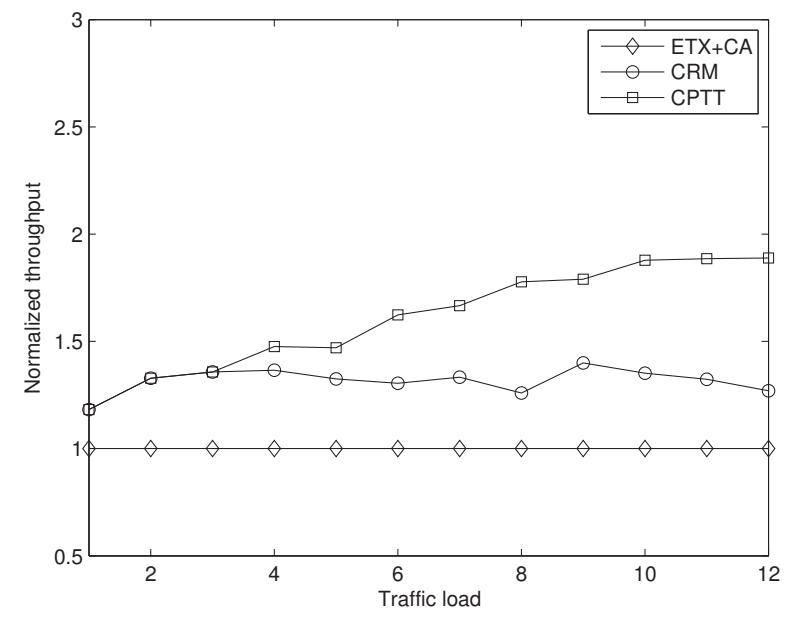

Fig. 4. Path throughput under different coding-aware routing metrics.

Fig. 4 illustrates the normalized throughput of the paths found by different routing metrics with respect to ETX+CA. It shows that $\mathrm{ETX}+\mathrm{CA}$ has the worst performance. The paths selected by CRM have higher throughput compared to those selected by ETX+CA since CRM considers more factors such as traffic load and interference. CPTT outperforms all the other coding-aware routing metrics, and the throughput of the paths discovered by CPTT is up to 1.9 times of the paths discovered with ETX+CA and 1.5 times of the paths discovered with CRM, respectively.

Fig. 5 shows the fraction of encoded traffic for ETX+CA, CRM and CPTT. Here, the fraction of encoded traffic is defined as the ratio of the traffic involved in network coding to the overall network throughput. As for ETX+CA, since it always selects path 1 , as the number of the injected flows increases, more and more traffic will be encoded at node 5. Therefore, the fraction of encoded traffic increases and reaches the maximum value of 5 units when the total traffic to be routed on path 1 is 5 units. After that, the traffic of the existing flow $1 \rightarrow 5 \rightarrow 2$ becomes the bottleneck for network coding and the fraction of encoded traffic starts to decrease. Considering the traffic load and interference, CRM will choose path 2 when path 1 is already congested. Thus, the fraction of encoded traffic for CRM is smaller than that for ETX+CA. CPTT could accurately quantify the effect of the factors such as traffic load, multirate, interference as well as network coding on path capacity and it has the minimum value of fraction of encoded traffic among all the coding-aware routing metrics.

\section{B. Evaluation on Random Topology}

In this subsection, we present the simulation results for a randomly generated network. We follows the simulation settings in [13], [14]: The nodes are randomly deployed in a square of side 400 unites. The transmission range of each node is set to 100 units, and the interference range is set to 200 units. The transmission rates can be chosen from 54,36,24 and $6 \mathrm{Mbps}$. We vary the number of existing flows and their

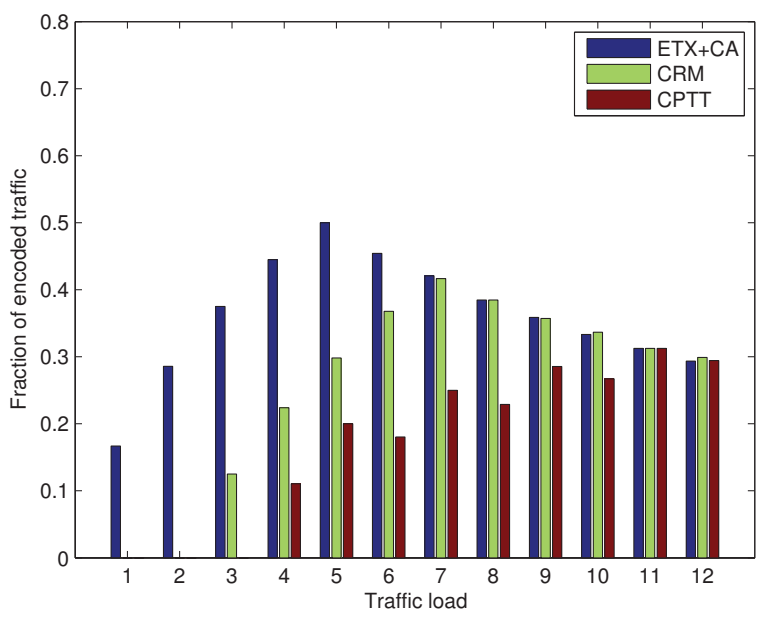

Fig. 5. Fraction of encoded traffic under different coding-aware routing metrics.

traffic rates, and compare the throughput of the paths selected by different coding-aware routing metrics.

Fig. 6 shows the comparison of path throughput normalized with respect to ETX+CA as the traffic load increases, from which similar conclusion can be drawn: The routes found by ETX+CA always have the minimum capacity. CRM works better than ETX+CA. The paths selected by CPTT achieve optimal end-to-end throughput, which is up to 2.1 times of the paths discovered under ETX+CA and 1.7 times of the paths discovered with CRM, respectively.

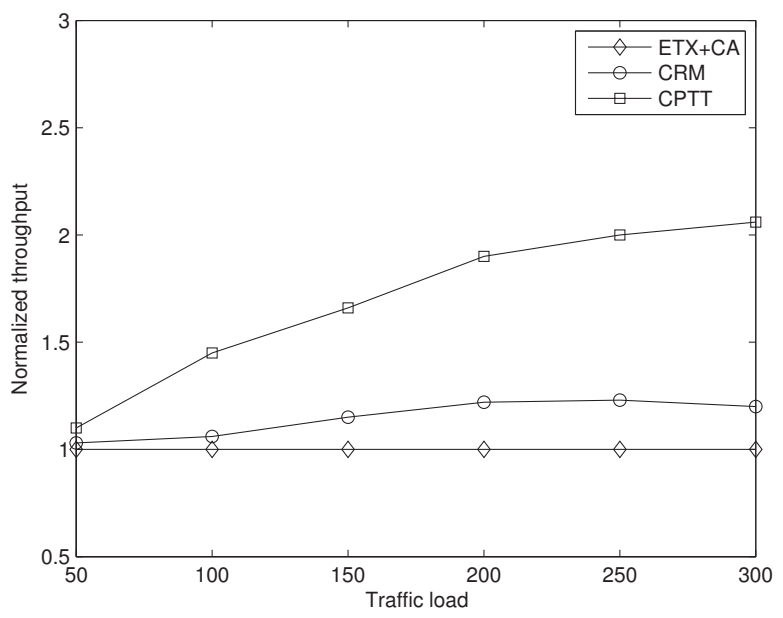

Fig. 6. Path throughput under different coding-aware routing metrics.

\section{CONCLUSION}

In this paper, we design a novel routing metric for path selection with network coding, which is called Coding-aware Path Transmission Time (CPTT). Based on the hyperlink conflict graph for both unicast and multicast, CPTT incorporates traffic load, multirate, transmission interference as well as network coding together in a unified manner, which could 
evaluate the performance of a path more accurately. Extensive simulations are conducted to demonstrate the effectiveness of CPTT. The results show that CPTT outperforms various existing coding-aware routing metrics and always discoveries paths with the highest throughput under network coding.

\section{REFERENCES}

[1] R. Ahlswede, N. Cai, S. R. Li, and R. W. Yeung, "Network information flow," IEEE Transactions on Information Theory, vol. 46, no. 4, pp. 1204-1216, July 2000.

[2] J. Liu, D. Goeckel, and D. Towsley, "Bounds on the gain of network coding and broadcasting in wireless networks," in Proc. of InfoCom 2007, Anchorage, Alaska, May 2007.

[3] J. Le, J. C. S. Liu, and D. Chiu, "How many packets can we encode? an analysis of practical wireless network coding," in Proc. of InfoCom 2008, Phoenix, AZ, April 2008.

[4] S. Katti, H. Rahul, W. Hu, D. Katabi, M. Medard, and J. Crowcroft, "Xors in the air: Practical wireless network coding," in Proc. of ACM SIGCOMM 2006, Pisa, Italy, September 2006.

[5] B. Ni, N. Santhapuri, Z. Zhong, and S. Nelakuditi, "Routing with opportunistically coded exchanges in wireless mesh networks," in Poster session of IEEE SECON 2006, Reston, VA, September 2006.

[6] J. Le, J. C. S. Liu, and D. Chiu, "DCAR: Distributed coding-aware routing in wireless networks," IEEE Transactions on Mobile Computing, vol. 9, no. 4, pp. 596-608, April 2010.

[7] Y. Wu, S. Das, K. Psounis, and R. Chandra, "Routing with a markovian metric to promote local mixing," in Proc. of InfoCom 2007, Anchorage, Alaska, May 2007.

[8] Y. Yan, Z. Zhao, B. Zhang, H. T. Mouftah, and J. Ma, "Rate-adaptive coding-aware multiple path routing for wireless mesh networks," in Proc. of Globecom 2008, New Orleans, LA, November 2008.

[9] Y. Peng, Y. Yang, X. Lu, and X. Ding, "Coding-aware routing for unicast sessions in multi-hop wireless networks," in Proc. of Globecom 2010, Miami, FL, December 2010.

[10] H. Zhai and Y. Fang, "Impact of routing metrics on path capacity in multirate and multihop wireless ad hoc networks," in Proc. of ICNP 2006, Santa Barbara, CA, November 2006.

[11] P. Gupta and P. R. Kumar, "The capacity of wireless networks," IEEE Transactions on Information Theory, vol. 46, no. 2, pp. 388-404, March 2000.

[12] D. Couto, D. Aguayo, J. Bicket, and R. Morris, "A high-throughput path metric for multi-hop wireless routing," in Proc. of ACM MobiCom 2003, San Diego, CA, September 2003.

[13] S. Sengupta, S. Rayanchu, and S. Banerjee, "An analysis of wireless network coding for unicast sessions: The case for coding-aware routing," in Proc. of InfoCom 2007, Anchorage, Alaska, May 2007.

[14] J. Zhang and Q. Zhang, "Cooperative network coding-aware routing for multi-rate wireless networks," in Proc. of InfoCom 2009, Rio de Janeiro, Brazil, April 2009. 